TRANSACTIONS OF THE

AMERICAN MATHEMATICAL SOCIETY

Volume 351, Number 12, Pages 5089-5106

S 0002-9947(99)02301-6

Article electronically published on May 21, 1999

\title{
*-POLYNOMIAL IDENTITIES OF MATRICES WITH THE TRANSPOSE INVOLUTION: THE LOW DEGREES
}

\author{
ALAIN D'AMOUR AND MICHEL RACINE
}

\begin{abstract}
In this paper, we investigate $*$-polynomial identities of minimal degree for the algebra of $n \times n$ matrices over a field, where $n<5$ and $*$ is the transpose involution. We first present some basic generators, and then proceed to show that all other minimal degree identities can be derived from those.
\end{abstract}

\section{INTRODUCTION}

Let $A$ be an associative algebra with involution $*$ over a field $F$. Amitsur [2] defined a $*$-polynomial to be a polynomial where the variables can also appear with a $*$; such polynomials can be evaluated on associative algebras with involution in the obvious way. When $A=M_{n}(F)$, the $n \times n$ matrices with entries in $F$, the ideal $I\left(M_{n}(F), *\right)$ of $*$-identities coincides with either $I\left(M_{n}(F), t\right)$ for $t$ the transpose involution or $I\left(M_{n}(F), s\right)$ for $s$ the symplectic involution ( $n$ even) [16, Thm. 3.1.62]. In both cases, the question of what is the minimal degree of a *-polynomial identity is still open. Giambruno [4, Thm. 1] has shown that

Theorem 1.1. Over a field $F$ of characteristic not 2 , if $f$ is a *-polynomial identity for $M_{n}(F)$ and $n>2$, then the degree of $f$ is greater than $n$.

While in general this result is not sharp, as we will see, it is for $n=3$ or 4 with * the transpose involution. The $*$-identities for $M_{2}(F), F$ of characteristic 0 , have been completely determined by Levchenko, Drensky and Giambruno [3], [7], [8] for both types of involutions. Our purpose is to determine the $*$-identities of minimal degree for $\left(M_{n}(F), t\right)$ when $n<5$. We have reason to believe that the situation for low $n$ 's is atypical and that it must be studied carefully before the general case can be dealt with.

There are two aspects to our results: producing the identities and showing that there are no others. The existence results hold for arbitrary fields and are proved using the fact that matrix algebras or some subspaces are in some sense of degree 1 or 2 . For the uniqueness results we must impose some mild characteristic restrictions, and these proofs ultimately depend on substitutions.

Our starting point is, as always, the standard polynomial

$$
\mathrm{S}_{n}\left(x_{1}, x_{2}, \ldots, x_{n}\right):=\sum_{\sigma \in \mathcal{S}_{n}}(-1)^{\sigma} x_{\sigma(1)} x_{\sigma(2)} \ldots x_{\sigma(n)},
$$

Received by the editors May 18, 1997.

1991 Mathematics Subject Classification. Primary 16R10, 16R50.

The second author's research is supported in part by a grant from NSERC. 
where the sum is taken over the symmetric group $\mathcal{S}_{n}$. Amitsur and Levitzki [1] have shown that $M_{n}(F)$ has no polynomial identity of degree less than $2 n$, that $\mathrm{S}_{2 n}$ is an identity of $M_{n}(F)$, and that if $|F|>2$ or $n>2$, then any identity of $M_{n}(F)$ of degree $2 n$ is a scalar multiple of $\mathrm{S}_{2 n}$. Let $H_{n}(F)$ denote the symmetric matrices and $K_{n}(F)=\left\{a-a^{t} \mid a \in M_{n}(F)\right\}$. If the characteristic of $F$ is not 2 then $K_{n}(F)$ is the set of skew-symmetric matrices, and when the characteristic is 2 , it coincides with the set of symmetric matrices with 0's along the diagonal. Since we are working over a fixed but arbitrary field, we will often use $M_{n}, H_{n}$ and $K_{n}$.

If the characteristic is not 2 , the existence of a $*$-identity of a given degree $d$ in an algebra $A$ is equivalent to the existence of a polynomial of the same degree which yields 0 for every substitution where the last $k$ variables are replaced by elements of $H(A, *)$ and the remaining $d-k$ variables are replaced by elements of $K(A, *)$, for $k$ some integer between 0 and $d$ (see for example [4]). Accordingly, we make the following

Definition 1.3. An $(n, d, s)$-identity is a multilinear $*$-polynomial identity of $M_{n}$

$$
f\left(z_{1}, \ldots, z_{d}\right)=\sum_{\sigma \in \mathcal{S}_{d}} \alpha_{\sigma} z_{\sigma(1)} \ldots z_{\sigma(d)},
$$

where $z_{i}=x_{i}-x_{i}^{*}$ for $1 \leq i \leq d-s$, and $z_{i}=x_{i}+x_{i}^{*}$ for $d-s+1 \leq i \leq d$.

Hence an $(n, d, s)$-identity is a multilinear identity of $M_{n}$ of degree $d$ with $s$ "symmetric" variables and $d-s$ "skew-symmetric" variables. Let

$$
\{a b c\}:=a b c+c b a, \quad \text { for } \quad a, b, c \in A .
$$

This triple product induces a Jordan triple system structure on $A, H(A, *)$, and $K(A, *)[11]$. Next, let

$$
[a, b]:=a b-b a, \quad a \circ b:=a b+b a, \quad a \mathrm{U}_{b}:=b a b .
$$

The Lie bracket induces a Lie algebra structure on $A$ and $K(A, *)$, and the circle product induces a Jordan algebra structure on $A$ and $H(A, *)$.

For a unital commutative ring $R$, the following identities are mentioned by Rowen in [16, Remark 2.5.15]:

$$
\begin{aligned}
x_{1}-x_{1}^{*} & \in I\left(M_{1}(R), t\right) ; \\
{\left[x_{1}-x_{1}^{*}, x_{2}-x_{2}^{*}\right] } & \in I\left(M_{2}(R), t\right) ; \\
{\left[\mathrm{S}_{3}\left(x_{1}-x_{1}^{*}, x_{2}-x_{2}^{*}, x_{3}-x_{3}^{*}\right), x_{4}\right] } & \in I\left(M_{3}(R), t\right) ; \\
\mathrm{S}_{6}\left(x_{1}-x_{1}^{*}, \ldots, x_{6}-x_{6}^{*}\right) & \in I\left(M_{4}(R), t\right) ; \\
{\left[x_{1}+x_{1}^{*}, x_{2}\right] } & \in I\left(M_{2}(R), s\right) ; \\
{\left.\left[x_{1}+x_{1}^{*}, x_{2}-x_{2}^{*}\right]^{2}, x_{3}\right] } & \in I\left(M_{4}(R), s\right) .
\end{aligned}
$$

Then Rowen cautiously adds: "As far as I know, all minimal identities of $\left(M_{n}(\mathbf{Z}), *\right)$ (for $n \leq 4$ ) are implied by these identities." As we will see below, more polynomials are required to obtain all the identities of minimal degree, at least in the case of the transpose involution.

\section{2. *-Identities of Minimal Degree for $M_{n}(F), n<5$}

In this section we exhibit some identities for $K_{n}(F), n<5$, and some $*$-identities for $M_{3}(F)$ involving one symmetric variable; in the next section we show that 
these suffice to obtain all minimal degree $*$-identities under some mild characteristic restrictions.

We recall that Kostant [5], [6] has shown that, for $n$ even, $\mathrm{S}_{2 n-2}$ is an identity of $K_{n}(F)$ but $\mathrm{S}_{2 n-3}$ isn't. This was extended to all $n$ 's by Rowen [15], [17]. Note that while this result says that $\mathrm{S}_{2 n-2}$ is the minimal degree standard identity satisfied by $K_{n}(F)$, it does not say that $K_{n}(F)$ satisfies no other identity of degree $2 n-2$ or less. This also implies that $\mathrm{S}_{2 n-2}\left(x_{1}-x_{1}^{*}, \ldots, x_{2 n-2}-x_{2 n-2}^{*}\right)$ is a $*$-polynomial identity of $M_{n}(F)$. Since the minimal degree of an identity of $H_{n}(F)$ is $2 n$ [10] and the minimal degree of an identity of $K_{n}(F)$ is less than or equal to $2 n-2$ [5], [6], [15], [17], the polynomial corresponding to $*$-identities of minimal degree must involve some "skew-symmetric variables". In view of some of Rowen's results, we expect most if not all of them to be skew.

We begin with $n=1$, in which case it is trivial to see that $\left(x_{1}-x_{1}^{*}\right) \in I\left(M_{1}\right)$. If $n=2, K_{2}(F)$ has dimension 1 and so

$$
\mathrm{S}_{2} \in I\left(K_{2}\right) \text {, and hence }\left[x_{1}-x_{1}^{*}, x_{2}-x_{2}^{*}\right] \in I\left(M_{2}\right) .
$$

We now turn to $n=3$. Let

$$
r\left(x_{1}, x_{2}, x_{3}, x_{4}\right):=\left[\mathrm{S}_{3}\left(x_{1}, x_{2}, x_{3}\right), x_{4}\right] .
$$

Since $\mathrm{S}_{3}$ is multilinear alternating of degree 3 and $K_{3}(F)$ has dimension 3 , it is easy to check that $\mathrm{S}_{3}$ is a central polynomial on $K_{3}$ (in fact it is an identity on $K_{3}$ in characteristic 2), and so $r\left(x_{1}, x_{2}, x_{3}, x_{4}\right) \in I\left(K_{3}\right)$. The dimensionality argument also shows that $\mathrm{S}_{4} \in I\left(K_{3}\right)$, a fact already known from the Kostant-Rowen results. Next, we point out that one could choose to view $K_{3}(F)$ as a Jordan triple system which happens to be isomorphic to $1 \times 3$ matrices over $F$ and is therefore of degree 1. But more directly, an easy computation yields

$$
\begin{aligned}
\mathrm{ABA}=-\left(a_{12} b_{12}+a_{13} b_{13}+a_{23} b_{23}\right) \mathrm{A}, \\
\text { for } \mathrm{A}=\left(a_{i j}\right), \quad \mathrm{B}=\left(b_{i j}\right) \in K_{3}(F) .
\end{aligned}
$$

Irrespective of one's point of view, (2.3) yields

$$
[\mathrm{ABA}, \mathrm{A}]=0, \quad \text { for } \mathrm{A}, \mathrm{B} \in K_{3}(F)
$$

and $[x y x, x]$ is an identity of $K_{3}$. To describe our next identity, we use tr to denote the trace and we linearize (2.3) to get

$$
\{\mathrm{A} \mathrm{B} \mathrm{C}\}=\frac{1}{2} \operatorname{tr}(\mathrm{AB}) \mathrm{C}+\frac{1}{2} \operatorname{tr}(\mathrm{CB}) \mathrm{A}, \quad \text { for } \quad \mathrm{A}, \mathrm{B}, \mathrm{C} \in K_{3}(F),
$$

where the half is purely formal and makes sense even in characteristic $2(\operatorname{tr}(\mathrm{AB})$ is a linear combination of terms whose coefficients are even integers). Now we consider

$$
p\left(x_{1}, x_{2}, x_{3}, x_{4}\right):=\sum_{(123)}\left\{x_{1}\left[x_{2}, x_{4}\right] x_{3}\right\},
$$

where $\sum_{(123)}$ denotes the sum over the cyclic permutations of $1,2,3$. If we assume that (2.4) holds formally, then $p\left(x_{1}, x_{2}, x_{3}, x_{4}\right)$ is a linear combination of $x_{1}, x_{2}$ and $x_{3}$ in which the coefficient of $x_{3}$ is

$$
\frac{1}{2}\left(\operatorname{tr}\left(x_{1}\left[x_{2}, x_{4}\right]\right)+\operatorname{tr}\left(x_{2}\left[x_{1}, x_{4}\right]\right)\right)=\frac{1}{2} \operatorname{tr}\left(\left[x_{1} x_{2}, x_{4}\right]\right)=0 .
$$


Since $p\left(x_{1}, x_{2}, x_{3}, x_{4}\right)$ is symmetric in $x_{1}, x_{2}$ and $x_{3}, p\left(x_{1}, x_{2}, x_{3}, x_{4}\right) \in I\left(K_{3}\right)$. Our last key polynomial for $K_{3}$ is

$$
\begin{aligned}
q\left(x_{1}, x_{2}, x_{3}, x_{4}\right):= & \sum_{(123)}\left\{x_{1}\left[x_{2}, x_{3}\right] x_{4}\right\}+\sum_{(124)}\left\{x_{1}\left[x_{2}, x_{4}\right] x_{3}\right\} \\
& +2\left(\left[x_{1}, x_{3}\right] \mathrm{D}_{x_{2}, x_{4}}+\left[x_{1}, x_{4}\right] \mathrm{D}_{x_{2}, x_{3}}\right. \\
& \left.-\left[x_{2}, x_{3}\right] \mathrm{D}_{x_{1}, x_{4}}-\left[x_{2}, x_{4}\right] \mathrm{D}_{x_{1}, x_{3}}\right)
\end{aligned}
$$

where $\mathrm{D}$ is the derivation

$$
x \mathrm{D}_{y, z}:=\left\{\begin{array}{lll}
x & y & z
\end{array}\right\}-\left\{\begin{array}{lll}
x & z & y
\end{array}\right\} .
$$

The polynomial $q$ is alternating in $x_{1}, x_{2}$ and symmetric in $x_{3}, x_{4}$. We claim that $q$ is an identity on $K_{3}$. Again assuming that (2.4) holds formally, we note that

$$
\begin{aligned}
2\left[x_{1}, x_{3}\right] \mathrm{D}_{x_{2}, x_{4}}= & \operatorname{tr}\left(\left[x_{1}, x_{3}\right] x_{2}\right) x_{4}+\operatorname{tr}\left(x_{4} x_{2}\right)\left[x_{1}, x_{3}\right] \\
& \quad-\operatorname{tr}\left(\left[x_{1}, x_{3}\right] x_{4}\right) x_{2}-\operatorname{tr}\left(x_{2} x_{4}\right)\left[x_{1}, x_{3}\right] \\
= & \operatorname{tr}\left(\left[x_{1}, x_{3}\right] x_{2}\right) x_{4}-\operatorname{tr}\left(\left[x_{1}, x_{3}\right] x_{4}\right) x_{2} .
\end{aligned}
$$

Therefore $q\left(x_{1}, x_{2}, x_{3}, x_{4}\right)$ is a linear combination of $x_{i}, 1 \leq i \leq 4$, and by symmetry we need only consider the coefficients of $x_{2}$ and $x_{4}$. The coefficient of $x_{2}$ is

$$
\begin{gathered}
\frac{1}{2}\left(\operatorname{tr}\left(x_{4}\left[x_{3}, x_{1}\right]\right)+\operatorname{tr}\left(x_{3}\left[x_{4}, x_{1}\right]\right)\right)-\operatorname{tr}\left(\left[x_{1}, x_{3}\right] x_{4}\right)-\operatorname{tr}\left(\left[x_{1}, x_{4}\right] x_{3}\right) \\
=\frac{1}{2} \operatorname{tr}\left(\left[x_{3} x_{4}, x_{1}\right]\right)-\operatorname{tr}\left(\left[x_{1}, x_{3} x_{4}\right]\right)=0 .
\end{gathered}
$$

The coefficient of $x_{4}$ is

$$
\begin{aligned}
\frac{1}{2}\left(\operatorname{tr}\left(x_{1}\left[x_{2}, x_{3}\right]\right)\right. & \left.+\operatorname{tr}\left(x_{2}\left[x_{3}, x_{1}\right]\right)+\operatorname{tr}\left(x_{3}\left[x_{1}, x_{2}\right]\right)+\operatorname{tr}\left(\left[x_{1}, x_{2}\right] x_{3}\right)\right) \\
& +\operatorname{tr}\left(\left[x_{1}, x_{3}\right] x_{2}\right)-\operatorname{tr}\left(\left[x_{2}, x_{3}\right] x_{1}\right)
\end{aligned}
$$

collecting terms, this equals

$$
\begin{aligned}
\frac{1}{2}\left(\operatorname{tr}\left(x_{1}\left[x_{2}, x_{3}\right]\right)\right. & \left.+\operatorname{tr}\left(x_{2}\left[x_{1}, x_{3}\right]\right)\right)+\operatorname{tr}\left(\left[x_{1}, x_{2}\right] x_{3}\right)-\operatorname{tr}\left(\left[x_{2}, x_{3}\right] x_{1}\right) \\
& =\frac{1}{2} \operatorname{tr}\left(\left[x_{1} x_{2}, x_{3}\right]\right)+\operatorname{tr}\left(\left[x_{1} x_{3}, x_{2}\right]\right)=0 .
\end{aligned}
$$

Hence $q\left(x_{1}, x_{2}, x_{3}, x_{4}\right) \in I\left(K_{3}\right)$, and we have proved

Proposition 2.7. The polynomials

$$
\begin{aligned}
p\left(x_{1}, x_{2}, x_{3}, x_{4}\right)= & \sum_{(123)}\left\{x_{1}\left[x_{2}, x_{4}\right] x_{3}\right\} \\
q\left(x_{1}, x_{2}, x_{3}, x_{4}\right)= & \sum_{(123)}\left\{x_{1}\left[x_{2}, x_{3}\right] x_{4}\right\}+\sum_{(124)}\left\{x_{1}\left[x_{2}, x_{4}\right] x_{3}\right\} \\
& +2\left(\left[x_{1}, x_{3}\right] \mathrm{D}_{x_{2}, x_{4}}+\left[x_{1}, x_{4}\right] \mathrm{D}_{x_{2}, x_{3}}\right. \\
& \left.\quad-\left[x_{2}, x_{3}\right] \mathrm{D}_{x_{1}, x_{4}}-\left[x_{2}, x_{4}\right] \mathrm{D}_{x_{1}, x_{3}}\right), \\
r\left(x_{1}, x_{2}, x_{3}, x_{4}\right)= & {\left[\mathrm{S}_{3}\left(x_{1}, x_{2}, x_{3}\right), x_{4}\right] }
\end{aligned}
$$

are identities of $K_{3}(F, t)$. 
There are a few more symmetry considerations worth noting about $p, q$ and $r$. For example, the polynomial $r$ is alternating in the first 3 variables. Hence, up to a sign, the only homogeneous polynomial obtainable from $r$ by allowing some of the variables to be equal is

$$
r\left(x_{1}, x_{2}, y, y\right)=-y^{2}\left[x_{1}, x_{2}\right]+y\left\langle x_{1} y x_{2}\right\rangle-\left\langle x_{1} y x_{2}\right\rangle y+\left[x_{1}, x_{2}\right] y^{2},
$$

in which we use the notation

$$
\langle a b c\rangle:=a b c-c b a, \quad \text { for } \quad a, b, c \in A .
$$

We also have

$$
\begin{gathered}
r\left(x_{1}, x_{2}, x_{3}, x_{4}\right)-r\left(x_{1}, x_{2}, x_{4}, x_{3}\right)-r\left(x_{1}, x_{4}, x_{3}, x_{2}\right)-r\left(x_{4}, x_{2}, x_{3}, x_{1}\right) \\
=2 \mathrm{~S}_{4}\left(x_{1}, x_{2}, x_{3}, x_{4}\right) .
\end{gathered}
$$

We already noted that the polynomial $q$ is alternating in $x_{1}$ and $x_{2}$ and symmetric in $x_{3}$ and $x_{4}$. One can also check that $q\left(x_{1}, y, y, y\right)=0$, while

$$
\begin{aligned}
q\left(x_{1}, x_{2}, y, y\right)=2 y^{2}[ & \left.x_{1}, x_{2}\right]+6 y\left\langle x_{1} y x_{2}\right\rangle-4 y\left[x_{1}, x_{2}\right] y \\
& +6\left\langle x_{1} y x_{2}\right\rangle y+2\left[x_{1}, x_{2}\right] y^{2}-8\left\langle x_{1} y^{2} x_{2}\right\rangle .
\end{aligned}
$$

Finally, by setting some of the variables equal in $p\left(x_{1}, x_{2}, x_{3}, x_{4}\right)$, we obtain

$$
p(y, y, x, y)=\{y[x, y] y\}=[\{y x y\}, y]=2[y x y, y]
$$

and

$$
\begin{array}{r}
p\left(y, y, x_{1}, x_{2}\right)-p\left(y, y, x_{2}, x_{1}\right)=-2 y^{2}\left[x_{1}, x_{2}\right]+2 y\left\langle x_{1} y x_{2}\right\rangle \\
+4 y\left[x_{1}, x_{2}\right] y+2\left\langle x_{1} y x_{2}\right\rangle y-2\left[x_{1}, x_{2}\right] y^{2} .
\end{array}
$$

Remarks 2.14. 1. The polynomials $p, q$ and $r$ generate an $F\left[\mathcal{S}_{4}\right]$-module under the action which permutes the variables. One can check that if the characteristic of $F$ is not 2 or 3 , then the character of this representation is $[3,1]+2[2,1,1]+[1,1,1,1]$, in the usual notation for Young diagrams.

2. In a certain sense, $[x y x, x] \in I\left(K_{3}\right)$ was already known. In [14, Thm. 2] it is shown that $\left(\operatorname{ad}_{x}\right)^{3}$ acts as a derivation on the Lie algebra $s l_{2}$ which is isomorphic to $K_{3}$. Expressing this as a polynomial identity of the adjoint representation yields $\left[\operatorname{ad}_{x} \operatorname{ad}_{y} \operatorname{ad}_{x}, \operatorname{ad}_{x}\right]$.

Consider now the polynomial

$$
g\left(x_{1}, x_{2}, x_{3}, x_{4}\right)=\sum_{\sigma \in \mathcal{S}_{2}}(-1)^{\sigma}\left(\left\{x_{\sigma(1)} x_{\sigma(2)}\left(x_{3} \circ x_{4}\right)\right\}-\left\{x_{\sigma(1)}\left(x_{\sigma(2)} \circ x_{4}\right) x_{3}\right\}\right),
$$

in which $x_{1}, x_{2}, x_{3}$ are skew-symmetric and $x_{4}$ is symmetric. Using $K_{n} \circ H_{n} \subset K_{n}$ and equation (2.4), one can verify that

$$
\begin{aligned}
g\left(x_{1}, x_{2}, x_{3}, x_{4}\right)=\frac{1}{2} & \left(\left(\operatorname{tr}\left(\left(x_{3} \circ x_{4}\right) x_{2}\right)-\operatorname{tr}\left(x_{3}\left(x_{2} \circ x_{4}\right)\right)\right) x_{1}\right. \\
& +\left(\operatorname{tr}\left(x_{3}\left(x_{1} \circ x_{4}\right)\right)-\operatorname{tr}\left(\left(x_{3} \circ x_{4}\right) x_{1}\right)\right) x_{2} \\
& +\left(\operatorname{tr}\left(x_{2}\left(x_{1} \circ x_{4}\right)\right)-\operatorname{tr}\left(x_{1}\left(x_{2} \circ x_{4}\right)\right)\right) x_{3} \\
& \left.\quad+\left(\operatorname{tr}\left(x_{1} x_{2}\right)-\operatorname{tr}\left(x_{2} x_{1}\right)\right)\left(x_{3} \circ x_{4}\right)\right) \\
=0 . &
\end{aligned}
$$

As was already mentioned, $\mathrm{S}_{3}\left(x_{1}, x_{2}, x_{3}\right.$ ) (for $x_{i} \in K_{3}$ ) is central in $M_{3}$ and therefore we have the following proposition. 
Proposition 2.16. The polynomials

$$
\begin{gathered}
g\left(x_{1}, x_{2}, x_{3}, x_{4}\right)=\sum_{\sigma \in \mathcal{S}_{2}}(-1)^{\sigma}\left(\left\{x_{\sigma(1)} x_{\sigma(2)}\left(x_{3} \circ x_{4}\right)\right\}\right. \\
\left.\quad-\left\{x_{\sigma(1)}\left(x_{\sigma(2)} \circ x_{4}\right) x_{3}\right\}\right), \\
r\left(x_{1}, x_{2}, x_{3}, x_{4}\right)=\left[\mathrm{S}_{3}\left(x_{1}, x_{2}, x_{3}\right), x_{4}\right]
\end{gathered}
$$

are $(3,4,1)$-identities.

Remark 2.17. Rowen [15] has shown that for $n$ odd, $\mathrm{S}_{2 n-2}$ is an $(n, 2 n-2,1)$ identity. An elementary proof for $n=3$ is given by the relation

$$
\mathrm{S}_{4}\left(x_{1}, x_{2}, x_{3}, x_{4}\right)=2 r\left(x_{1}, x_{2}, x_{3}, x_{4}\right)-h\left(x_{1}, x_{2}, x_{3}, x_{4}\right),
$$

where

$$
h\left(x_{1}, x_{2}, x_{3}, x_{4}\right)=\sum_{(123)}\left(x_{4} \circ x_{3}\right) D_{x_{2}, x_{1}} .
$$

To see that $h$ is an identity, it suffices, by symmetry considerations, to show that the coefficients of $x_{3} \circ x_{4}$ and $x_{3}$ vanish; the former is $\frac{1}{2}\left(\operatorname{tr}\left(x_{1} x_{2}\right)-\operatorname{tr}\left(x_{2} x_{1}\right)\right)=0$ and the latter is $\frac{1}{2}\left(\operatorname{tr}\left(\left(x_{2} \circ x_{4}\right) x_{1}\right)-\operatorname{tr}\left(\left(x_{1} \circ x_{4}\right) x_{2}\right)\right)=0$.

We now handle $n=4$. We will later see that in this case, there are no mixed identities and so we only need to examine $K_{4}$. In order to present $K_{4}$ 's identities in their proper context, we first recall a general construction of a "degree 2" Jordan triple system (see [11]). Let $L$ be an extension of $F$ with involution ${ }^{-}$, that is an $F$-linear automorphism of period $2, V$ an $L$-module, $Q: V \rightarrow L$ a quadratic form and $s: V \rightarrow V$ an $L$-linear map satisfying

$$
s^{2}=\mathrm{Id}, \quad s(c v)=\bar{c} s(v) \text { and } Q(s v)=\overline{Q(v)}, \quad \text { for all } c \in L, v \in V .
$$

Then

$$
\{u v w\}:=Q(u, s v) w+Q(w, s v) u-Q(u, w) s v
$$

(where $Q(u, v)$ is the bilinearization of $Q$ ) defines a Jordan triple product on $V$. Such Jordan triple systems are referred to as quadratic form triples [11, Example 1.6]. By (2.18),

$$
Q(s u, v)=\overline{Q(s s u, s v)}=\overline{Q(u, s v)} .
$$

In particular let $L=F$ (so - is the identity map) and $V=K_{4}(F)$. The Pfaffian [11] is a nondegenerate quadratic form on $K_{4}$; it is a square root of the determinant and so is determined up to sign. We make the following choice:

$$
\text { Pf } \mathrm{A}=a_{12} a_{34}-a_{13} a_{24}+a_{14} a_{23}, \quad \text { for } \mathrm{A}=\left[\begin{array}{cccc}
0 & a_{12} & a_{13} & a_{14} \\
-a_{12} & 0 & a_{23} & a_{24} \\
-a_{13} & -a_{23} & 0 & a_{34} \\
-a_{14} & -a_{24} & -a_{34} & 0
\end{array}\right] \text {. }
$$

For $\mathrm{A}$ as above, define the following involutory map on $K_{4}$ :

$$
s \mathrm{~A}=\left[\begin{array}{cccc}
0 & -a_{34} & a_{24} & -a_{23} \\
a_{34} & 0 & -a_{14} & a_{13} \\
-a_{24} & a_{14} & 0 & -a_{12} \\
a_{23} & -a_{13} & a_{12} & 0
\end{array}\right]
$$


One checks that

$$
\mathrm{ABA}=\operatorname{Pf}(\mathrm{A}, s \mathrm{~B}) \mathrm{A}-\operatorname{Pf}(\mathrm{A}) s \mathrm{~B},
$$

where $\operatorname{Pf}(\mathrm{A}, \mathrm{B})$ is the bilinearization of the Pfaffian. Therefore $K_{4}$ with the usual triple product is a quadratic form triple. The Jordan nature of $K_{4}$ prompts us to revisit the polynomial

$$
\kappa\left(x_{1}, x_{2}, x_{3}, y\right):=y^{2} \mathrm{~S}_{3}\left(\mathrm{~V}_{x_{1}}, \mathrm{~V}_{x_{2}}, \mathrm{~V}_{x_{3}}\right)-\left(y \mathrm{~S}_{3}\left(\mathrm{~V}_{x_{1}}, \mathrm{~V}_{x_{2}}, \mathrm{~V}_{x_{3}}\right)\right) \circ y
$$

(where $y \mathrm{~V}_{x}:=y \circ x$ ), which has been shown (by the second author in [13]) to be an identity for all Jordan algebras of degree 2. The expression in (2.21) can be rewritten in associative terms,

$$
\kappa\left(x_{1}, x_{2}, x_{3}, y\right)=\mathrm{S}_{4}\left(x_{1}, x_{2}, x_{3}, y^{2}\right)-y \circ \mathrm{S}_{4}\left(x_{1}, x_{2}, x_{3}, y\right)
$$

or in terms of linear triple products,

$$
\begin{aligned}
\kappa\left(x_{1}, x_{2}, x_{3}, y\right):=\sum_{\sigma \in \mathcal{S}_{3}}(-1)^{\sigma}\left(\left\{\left\{y y x_{\sigma(1)}\right\} x_{\sigma(2)} x_{\sigma(3)}\right\}\right. \\
-\left\{\left\{y x_{\sigma(1)} x_{\sigma(2)}\right\} x_{\sigma(3)} y\right\} \\
\left.-\left\{x_{\sigma(1)}\left\{y x_{\sigma(2)} x_{\sigma(3)}\right\} y\right\}\right),
\end{aligned}
$$

and we denote its linearization by

$$
\begin{aligned}
\kappa\left(x_{1}, x_{2}, x_{3} ; x ; y\right):= & (x \circ y) \mathrm{S}_{3}\left(\mathrm{~V}_{x_{1}}, \mathrm{~V}_{x_{2}}, \mathrm{~V}_{x_{3}}\right)-\left(x \mathrm{~S}_{3}\left(\mathrm{~V}_{x_{1}}, \mathrm{~V}_{x_{2}}, \mathrm{~V}_{x_{3}}\right)\right) \circ y \\
& \left(y \mathrm{~S}_{3}\left(\mathrm{~V}_{x_{1}}, \mathrm{~V}_{x_{2}}, \mathrm{~V}_{x_{3}}\right)\right) \circ x .
\end{aligned}
$$

The key reason why $\kappa$ is an identity for degree 2 Jordan algebras is that an alternating sum vanishes whenever two of the alternating variables are also symmetric. On the other hand, if $\kappa$ is evaluated on a quadratic form triple system, then the result is a linear combination of $y, s y, x_{\sigma(i)}$ and $s x_{\sigma(i)}, 1 \leq i \leq 3$. Starting with (2.23) and using (2.18) and (2.19), the coefficients of $y$ in the first, second and third terms of $\kappa$ are respectively given by

$$
\sum_{\sigma \in \mathcal{S}_{3}}(-1)^{\sigma} Q\left(x_{\sigma(1)}, s y\right) Q\left(x_{\sigma(3)}, s x_{\sigma(2)}\right),
$$

$$
\begin{gathered}
-\sum_{\sigma \in \mathcal{S}_{3}}(-1)^{\sigma}\left(Q\left(x_{\sigma(2)}, s x_{\sigma(3)}\right) Q\left(y, s x_{\sigma(1)}\right)+2 Q\left(y, s x_{\sigma(3)}\right) Q\left(x_{\sigma(2)}, s x_{\sigma(1)}\right)\right. \\
\left.-Q\left(s x_{\sigma(1)}, s x_{\sigma(3)}\right) Q\left(y, x_{\sigma(2)}\right)\right),
\end{gathered}
$$

and

$$
\begin{gathered}
-\sum_{\sigma \in \mathcal{S}_{3}}(-1)^{\sigma}\left(Q\left(x_{\sigma(1)}, s y\right) \overline{Q\left(x_{\sigma(3)}, s x_{\sigma(2)}\right)}+Q\left(x_{\sigma(1)}, s x_{\sigma(3)}\right) \overline{Q\left(y, s x_{\sigma(2)}\right)}\right. \\
\left.-Q\left(x_{\sigma(1)}, x_{\sigma(2)}\right) \overline{Q\left(y, x_{\sigma(3)}\right)}\right)
\end{gathered}
$$

The third summands of (2.26) and (2.27) are alternating sums of symmetric functions and therefore 0. By (2.20),

$$
Q\left(x_{\sigma(1)}, s y\right) \overline{Q\left(x_{\sigma(3)}, s x_{\sigma(2)}\right)}=Q\left(s x_{\sigma(3)}, x_{\sigma(2)}\right) \overline{Q\left(s x_{\sigma(1)}, y\right)},
$$


and so the remaining two terms of (2.27) differ only by a transposition and their alternating sum cancels. Similarly the first term of (2.26) cancels with half the second term, and the coefficient of $y$ reduces to

$$
\begin{gathered}
\sum_{\sigma \in \mathcal{S}_{3}}(-1)^{\sigma}\left(Q\left(x_{\sigma(1)}, s y\right) Q\left(x_{\sigma(3)}, s x_{\sigma(2)}\right)-Q\left(y, s x_{\sigma(3)}\right) Q\left(x_{\sigma(2)}, s x_{\sigma(1)}\right)\right) \\
=\sum_{\sigma \in \mathcal{S}_{3}}(-1)^{\sigma} Q\left(x_{\sigma(2)}, s x_{\sigma(1)}\right)\left(Q\left(s y, x_{\sigma(3)}\right)-\overline{Q\left(s y, x_{\sigma(3)}\right)}\right) .
\end{gathered}
$$

Similarly the coefficient of $s y$ is given by

$$
\sum_{\sigma \in \mathcal{S}_{3}}(-1)^{\sigma} Q\left(y, x_{\sigma(1)}\right)\left(Q\left(s x_{\sigma(3)}, x_{\sigma(2)}\right)-\overline{Q\left(s x_{\sigma(3)}, x_{\sigma(2)}\right)}\right) .
$$

As the following example will show, the expressions (2.28) and (2.29) are in general not identically 0 .

Example 2.30. Let $L=F[\theta]$, a quadratic extension of a field $F$ of characteristic not 2 with canonical involution $\bar{\theta}=-\theta, V$ the direct sum of three hyperbolic planes spanned by hyperbolic pairs $u_{i}, v_{i}, 1 \leq i \leq 3$, and $s: V \rightarrow V$ the $L$-semilinear map given by $s u_{1}=u_{1}, s v_{1}=v_{1}, s u_{2}=u_{3}, s v_{2}=v_{3}, s u_{3}=u_{2}$ and $s v_{3}=v_{2}$. For $x_{1}=u_{1}, x_{2}=\theta v_{1}, x_{3}=u_{3}$ and $y=\theta v_{2}$, by (2.28), the coefficient of $v_{2}$ in $\kappa\left(x_{1}, x_{2}, x_{3}, y\right)$ is

$$
\begin{aligned}
\left(Q\left(u_{1}, \theta v_{1}\right)(\right. & \left.Q\left(u_{3},-\theta v_{3}\right)-\overline{Q\left(u_{3},-\theta v_{3}\right)}\right) \\
& \left.-Q\left(-\theta v_{1}, u_{1}\right)\left(Q\left(u_{3},-\theta v_{3}\right)-\overline{Q\left(u_{3},-\theta v_{3}\right)}\right)\right) \theta \\
= & -4 \theta^{3} .
\end{aligned}
$$

Therefore $\kappa$ is not an identity for arbitrary Jordan triple systems of a quadratic form. However, we will prove

Theorem 2.31. If $(V, Q,-)$ is a Jordan triple system of a quadratic form $Q$ and - is the identity map, then $\kappa\left(x_{1}, x_{2}, x_{3}, y\right)$ is a polynomial identity of $V$.

Proof. If - is the identity map then (2.20) becomes

$$
Q(s u, v)=Q(u, s v)
$$

and $Q(s u, v)$ is symmetric in $u$ and $v$. Therefore the alternating sums in (2.28) and (2.29) vanish. Since $\kappa$ is alternating in $x_{1}, x_{2}, x_{3}$, it is enough to show that the coefficients of $x_{3}$ and $s x_{3}$ both vanish. Arguing as above, the coefficient of $x_{3}$ in $\kappa\left(x_{1}, x_{2}, x_{3}, y\right)$ is

$$
3 \sum_{\sigma \in \mathcal{S}_{2}}(-1)^{\sigma} Q\left(x_{\sigma(1)}, s x_{\sigma(2)}\right) Q(y, s y),
$$

which is 0 , while the coefficient of $s x_{3}$ is

$$
\begin{array}{r}
\sum_{\sigma \in \mathcal{S}_{2}}(-1)^{\sigma}\left(Q\left(x_{\sigma(2)}, y\right)\left(Q\left(x_{\sigma(1)}, s y\right)-\overline{Q\left(x_{\sigma(1)}, s y\right)}\right)\right. \\
\left.+Q\left(s x_{\sigma(1)}, x_{\sigma(2)}\right) Q(y, y)\right),
\end{array}
$$

which is also 0 .

Corollary 2.32. $\kappa\left(x_{1}, x_{2}, x_{3}, y\right)$ is a polynomial identity of $K_{4}(F, t)$. 
Remark 2.33. The polynomials

$$
\kappa_{i j}:=\kappa\left(x_{1}, \ldots, \widehat{x_{i}}, \ldots, \widehat{x_{j}}, \ldots, x_{5} ; x_{i} ; x_{j}\right), \quad 1 \leq i<j \leq 5,
$$

generate $I\left(K_{4}\right)$ as $F\left[\mathcal{S}_{5}\right]$-module, and the dimension is 10 . One can check that if the characteristic of $F$ is not 2 or 3 , the character of this representation is $[3,1,1]$ $+[2,1,1,1]$ in the usual notation for Young diagrams.

\section{UNIQUENESS}

We now proceed to show that under some restrictions on the base field, any *-polynomial identity of $M_{n}$ of minimal degree for $n<5$ can be obtained from the identities presented in the previous section. We recall that whenever the degree of each variable $x_{i}$ in an identity $p\left(x_{1}, \ldots, x_{m}\right)$ is less than $|F|$, then each homogeneous component of $p$ is also an identity. Accordingly, we will carry out a case by case study of homogeneous *-PI's. In order to simplify our calculations, we first introduce a useful result of Ma.

In [9], Ma defines a partial ordering on the homogeneous elements of the free associative algebra $F[X]$. A polynomial $p\left(x_{1}, \ldots, x_{m}\right)$ is said to be of type $\left[n_{1}, \ldots, n_{m}\right]$ if the $n_{j}$ 's are the degrees of the $x_{i}$ 's rearranged in nonincreasing order. (If an integer is repeated $k$ times, we denote this with an exponent; for example, $\left[3,2^{2}, 1^{3}\right]$ means $[3,2,2,1,1,1]$.) Given homogeneous polynomials $p$ and $p^{\prime}$ of degree $n$ and $n^{\prime}$ and of type $\left[n_{1}, \ldots, n_{m}\right]$ and $\left[n_{1}^{\prime}, \ldots, n_{m}^{\prime}\right]$ respectively, we say that $p$ is lower than $p^{\prime}$ in the partial ordering if and only if either (i) $n<n^{\prime}$, or (ii) $n=n^{\prime}$ and $n_{j}>n_{j}^{\prime}$ for the first $j$ such that $n_{j} \neq n_{j}^{\prime}$. Otherwise two polynomials are not comparable.

Now let $f\left(x_{1}, \ldots, x_{r}, y_{1}, \ldots, y_{s}, \ldots, z_{1}, \ldots, z_{t}\right)$ be a homogeneous polynomial identity of type $\left[m^{r}, n^{s}, \ldots, u^{t}\right]$ on some subspace $V$ of $M_{n}(F)$ (e.g. $V=H_{n}$ or $K_{n}$ ) and set $m_{0}=\max \{m, n, \ldots, u, r, s, \ldots, t\}$. The following theorem provides a relation of symmetry between variables of equal degree in $f$, depending on how many there are.

Theorem 3.1. If the characteristic of $F$ does not divide $m_{0}$ ! and $|F|>2 m_{0}-1$, then $f=f_{0}+f_{1}$, where the $f_{i}, i=0,1$, are identities of the same type as $f$ and for each $k$ with $0<k \leq m_{0}, f_{0}$ is symmetric or skew-symmetric in all variables of degree $k$, depending on whether $k$ is even or odd, while $f_{1}$ comes from identities of lower type.

We can outline Ma's proof as follows. One first establishes the above result for a pair of variables $x, y$ of some given degree $m$ in $f$; then, by acting on all $r$ variables of that same degree $m$ with the symmetric group $\mathcal{S}_{r}$, one obtains the desired symmetry property among those particular $r$ variables. Repeating the procedure for each degree separately yields the general result.

It becomes apparent that we can extend this result to $(n, d, s)$-identities by fixing the degree, keeping the $s$ symmetric and $d-s$ skew-symmetric variables apart, and acting on them via $\mathcal{S}_{s} \times \mathcal{S}_{d-s}$. Thus, when considering a typical homogeneous $(n, d, s)$-identity, we may assume the symmetry properties of Theorem 3.1, and with this, reduce the number of arbitrary coefficients involved in our calculations.

The case $n=1$ being trivial $\left(K_{n}=\{0\}\right.$, so $x-x^{*}$ is a $*$-identity), we begin with $n=2$. If at least one of the variables (say $\left.x_{2}\right)$ is symmetric in $f\left(x_{1}, x_{2}\right)=$ $\alpha x_{1} x_{2}+\beta x_{2} x_{1}$, then $f\left(e_{12} \pm e_{21}, e_{22}\right)=\alpha e_{12} \pm \beta e_{21}=0$ implies $\alpha=\beta=0$; hence, the $*$-identities of $M_{2}$ of minimal degree do not involve any symmetric variables. Now $f\left(e_{12}-e_{21}, e_{12}-e_{21}\right)=0$ implies $\alpha=-\beta$, and this with (2.1) proves 
Theorem 3.2. If $|F|>2$, then any polynomial identity of $K_{2}(F, t)$ of minimal degree is a scalar multiple of $\mathrm{S}_{2}\left(x_{1}, x_{2}\right)$, and any *-polynomial identity of $\left(M_{2}(F), t\right)$ of minimal degree is a consequence of $\mathrm{S}_{2}\left(x_{1}-x_{1}^{*}, x_{2}-x_{2}^{*}\right)$.

Remark 3.3. Although not of minimal degree, it is not hard to check that the polynomials $\left[x_{1}, x_{2}\right] x_{3}, x_{3}\left[x_{1}, x_{2}\right], x_{1} x_{2} x_{3}-x_{3} x_{2} x_{1}$ and $x_{1} x_{3} x_{2}-x_{2} x_{3} x_{1}$ form a basis for the space of $(2,3,1)$-identities.

For $n=3$ and 4 , the minimal degree of a $*$-identity is $n+1$. In these cases, the next lemma provides an upper bound on the possible number of symmetric variables involved.

Lemma 3.4. For $n \geq 2$ and $*=t$, an $(n, n+1, s)$-identity must have $s \leq 1$.

Proof. Suppose $f\left(z_{1}, \ldots, z_{n+1}\right)=\sum_{\sigma \in \mathcal{S}_{n+1}} \alpha_{\sigma} z_{\sigma(1)} \ldots z_{\sigma(n+1)} \neq 0$ is an $(n, n+1, s)$ identity. We first show that $s \leq 2$, and if $s=2$, then the two symmetric variables are adjacent in each and every monomial of $f$. For, suppose some monomial of $f$ has two non-adjacent symmetric variables (e.g. when $s \geq 3$ ); renaming the variables and scaling if necessary, we may assume this monomial to be $z_{1} z_{2} \ldots z_{n+1}$ (with $\alpha_{1}=1$ ) where say $z_{i}$ and $z_{j}$ are symmetric, $j>i+1$. Set

$$
\begin{aligned}
& A_{k}=e_{k k+1} \pm e_{k+1 k}, \quad 1 \leq k \leq i-1, \quad A_{i}=e_{i i}, \\
& A_{k}=e_{k-1 k} \pm e_{k k-1}, \quad i+1 \leq k \leq j-1, \quad A_{j}=e_{j-1 j-1}, \\
& A_{k}=e_{k-2 k-1} \pm e_{k-1 k-2}, \quad j+1 \leq k \leq n+1,
\end{aligned}
$$

where we take + or - in $A_{k}$ according as $z=x_{i}+x_{i}^{*}$ or $z=x_{i}-x_{i}^{*}$. Then

$$
f\left(A_{1}, \ldots, A_{n+1}\right)=e_{1 n}+\sum_{\substack{m \neq 1 \\ r \neq n}} \alpha_{m r} e_{m r} \neq 0
$$

a contradiction.

Next we point out that if $s=2$ then $f$ cannot be alternating in its symmetric variables $z_{n}, z_{n+1}$, for otherwise, setting $y_{i}=z_{i}, 1 \leq i \leq n-1, y_{n}=\left[z_{n}, z_{n+1}\right]$, we obtain $f\left(y_{1}, \ldots, y_{n}\right) \in I\left(K_{n}\right)$ (using $\left.K_{n}=\operatorname{Span}\left(\left[H_{n}, H_{n}\right]\right)\right)$, which contradicts Giambruno's Theorem 1.1. Hence, $p\left(z_{1}, \ldots, z_{n-1}, y\right):=f\left(z_{1}, \ldots, z_{n-1}, y, y\right) \neq 0$ is a *-polynomial identity of $M_{n}$ whose monomials are of the form

$$
z_{\sigma(1)} \ldots z_{\sigma(i-1)} y^{2} z_{\sigma(i)} \ldots z_{\sigma(n-1)}
$$

where $y$ is symmetric and $z_{j}$ are skew-symmetric. Assuming that $\alpha_{1}=1$ and that $y$ occurs in the $i^{\text {th }}$ position, set $A_{j}=e_{j j+1}-e_{j+1 j}, B=e_{i i}$; then $p\left(A_{1}, \ldots, A_{n-1}, B\right)=$ $e_{1 n}+\cdots \neq 0$, again yielding a contradiction. Thus $s \leq 1$.

Consider now the case $n=3$. By (1.1), there are no $(3, d, s)$-identities for $d<4$, and by lemma 3.4, $(3,4, s)$-identities must have $s \leq 1$. Suppose first that $s=0$, that is, we look at the identities of $K_{3}$. According to (3.1), we need only consider homogeneous polynomials of degree [4], [3,1], [2,2], [2,1,1] and $[1,1,1,1]$ with the third polynomial symmetric in its 2 variables, the fourth alternating in its linear variables, and the last alternating in all its variables. The $[1,1,1,1]$ polynomials are multiples of $\mathrm{S}_{4}$, and so by (2.10) they are a consequence of $r$. Since $x^{4} \notin I\left(K_{3}\right)$, we are left with the middle three types.

An arbitrary polynomial of type $[3,1]$ has the form

$$
f(x, y)=\alpha_{1} x y^{3}+\alpha_{2} y x y^{2}+\alpha_{3} y^{2} x y+\alpha_{4} y^{3} x .
$$


Assume that $f \in I\left(K_{3}\right)$. Letting $x=e_{12}-e_{21}$ and $y=e_{23}-e_{32}$ yields $\alpha_{1}=0=$ $\alpha_{4}$. Letting $y=x$ and using the fact that $x^{4} \notin I\left(K_{3}\right)$ yields $\alpha_{3}=-\alpha_{2}$. Therefore

$$
f(x, y)=\alpha[y x y, y]
$$

which, by (2.12), is a consequence of $p$.

Next, a polynomial of type [2,2] symmetric in its 2 variables has the form

$$
f(x, y)=\alpha_{1}\left(x^{2} y^{2}+y^{2} x^{2}\right)+\alpha_{2}(x y x y+y x y x)+\alpha_{3}\left(x y^{2} x+y x^{2} y\right) .
$$

Assume that $f \in I\left(K_{3}\right)$ and substitute $x=e_{12}-e_{21}, y=e_{23}-e_{32}$. This yields $\alpha_{3}=0=\alpha_{1}$. As in the previous case, letting $y=x$ yields $\alpha_{2}=0$. Thus $K_{3}$ has no identity of type $[2,2]$ which is symmetric in its variables.

An arbitrary polynomial of type $[2,1,1]$ which is alternating in its degree 1 variables can be written (recall the notation (2.24))

$$
\begin{aligned}
f\left(x_{1}, x_{2}, y\right)=\alpha_{1} y^{2}\left[x_{1}, x_{2}\right]+\alpha_{2} y\left\langle x_{1} y x_{2}\right\rangle+\alpha_{3} y\left[x_{1}, x_{2}\right] y \\
+\alpha_{4}\left\langle x_{1} y x_{2}\right\rangle y+\alpha_{5}\left[x_{1}, x_{2}\right] y^{2}+\alpha_{6}\left\langle x_{1} y^{2} x_{2}\right\rangle .
\end{aligned}
$$

Assume that $f \in I\left(K_{3}\right)$. We wish to simplify $f$ by adding known identities to it. By (2.11), subtracting a multiple of $q\left(x_{1}, x_{2}, y, y\right)$, we may assume that $\alpha_{6}=0$. Since identities of $K_{3}$ of type [3,1] have no terms of the form $x_{1} y^{3}$ or $y^{3} x_{1}$, letting $x_{2}=y$ yields

$$
\alpha_{1}+\alpha_{2}=0, \quad \alpha_{4}+\alpha_{5}=0
$$

and

$$
f\left(x_{1}, y, y\right)=\left(\alpha_{2}+\alpha_{3}-\alpha_{5}\right)\left[y x_{1} y, y\right] .
$$

By (2.12), subtracting a multiple of $p\left(y, y, x_{1}, y\right)$ from $f\left(x_{1}, y, y\right)$, we may assume that

$$
\alpha_{2}+\alpha_{3}-\alpha_{5}=0
$$

and

$$
\begin{gathered}
f\left(x_{1}, x_{2}, y\right)=\alpha_{1}\left(y^{2}\left[x_{1}, x_{2}\right]-y\left\langle x_{1} y x_{2}\right\rangle\right)+\left(\alpha_{1}-\alpha_{4}\right) y\left[x_{1}, x_{2}\right] y \\
+\alpha_{4}\left(\left\langle x_{1} y x_{2}\right\rangle y-\left[x_{1}, x_{2}\right] y^{2}\right) .
\end{gathered}
$$

By (2.8), adding $\alpha_{4} r\left(x_{1}, x_{2}, y, y\right)$, we may assume that

$$
f\left(x_{1}, x_{2}, y\right)=\alpha\left(y^{2}\left[x_{1}, x_{2}\right]-y\left\langle x_{1} y x_{2}\right\rangle+y\left[x_{1}, x_{2}\right] y\right) .
$$

Finally, substituting $x_{1}=e_{12}-e_{21}, x_{2}=e_{23}-e_{32}$ and $y=e_{13}-e_{31}$ yields $\alpha=0$. Hence $f$ is a consequence of $p, q$ and $r$.

We now turn to the case $s=1$ and examine (3,4,1)-identities. Once again, we use (3.1) and concentrate our efforts on homogeneous polynomials of degree 4 of various types. Here the types [4] and [2,2] aren't relevant. A typical polynomial of type $[3,1]$ has the form

$$
f(x, y)=\alpha_{1} x^{3} y+\alpha_{2} x^{2} y x+\alpha_{3} x y x^{2}+\alpha_{4} y x^{3},
$$

and we let $x \in K_{3}, y \in H_{3}$. Setting $x=e_{12}-e_{21}, y=e_{22}$ yields $\alpha_{1}+\alpha_{3}=0=$ $\alpha_{2}+\alpha_{4}$, while the substitution $x=e_{12}-e_{21}, y=e_{23}-e_{32}$ implies $\alpha_{1}=\alpha_{4}=0$. Therefore, $f \equiv 0$. 
Next we let

$$
\begin{aligned}
f\left(y, x_{1}, x_{2}\right) & =\alpha_{1} y^{2} x_{1} x_{2}+\alpha_{2} y^{2} x_{2} x_{1}+\alpha_{3} y x_{1} y x_{2}+\alpha_{4} y x_{2} y x_{1}+\alpha_{5} y x_{1} x_{2} y \\
& +\alpha_{6} y x_{2} x_{1} y+\alpha_{7} x_{1} y x_{2} y+\alpha_{8} x_{2} y x_{1} y+\alpha_{9} x_{1} x_{2} y^{2}+\alpha_{10} x_{2} x_{1} y^{2} \\
& +\alpha_{11} x_{1} y^{2} x_{2}+\alpha_{12} x_{2} y^{2} x_{1}
\end{aligned}
$$

be an arbitrary $(3,4,1)$-identity of type $[2,1,1], y, x_{1} \in K_{3}, x_{2} \in H_{3}$. We use the following four substitutions:

$$
\begin{gathered}
x_{1}=e_{12}-e_{21}, x_{2}=e_{11}, y=e_{23}-e_{32} \Rightarrow \alpha_{1}=\alpha_{10}=0, \\
x_{1}=e_{23}-e_{32}, x_{2}=e_{11}, y=e_{12}-e_{21} \Rightarrow \alpha_{4}=\alpha_{7}=0, \\
x_{1}=e_{12}-e_{21}, x_{2}=e_{22}, y=e_{23}-e_{32} \Rightarrow \alpha_{2}=-\alpha_{12} \text { and } \alpha_{9}=-\alpha_{11}, \\
x_{1}=e_{23}-e_{32}, x_{2}=e_{23}+e_{32}, y=e_{12}-e_{21} \\
\Rightarrow \alpha_{5}=\alpha_{6}, \alpha_{2}=\alpha_{9}, \text { and } \alpha_{11}=\alpha_{12} .
\end{gathered}
$$

Putting all these together, we conclude that $f$ has the form

$$
\begin{aligned}
f\left(y, x_{1}, x_{2}\right)= & \alpha\left(y^{2} x_{2} x_{1}+x_{1} x_{2} y^{2}-x_{1} y^{2} x_{2}-x_{2} y^{2} x_{1}\right)+\beta y x_{1} y x_{2} \\
& +\gamma\left(y x_{1} x_{2} y+y x_{2} x_{1} y\right)+\delta x_{2} y x_{1} y .
\end{aligned}
$$

Since there are no identities of type [3,1], the equation $f\left(y, y, x_{2}\right)=0$ gives $\alpha=$ $\beta=-\gamma=\delta$; we then observe that $f\left(y, x_{1}, x_{2}\right)$ is a scalar multiple of $g\left(y, x_{1}, y, x_{2}\right)$.

Finally, by $(3.1)$ we may assume that a $(3,4,1)$-identity of type $[1,1,1,1]$ has the form

$$
\begin{aligned}
f\left(x_{1}, x_{2}, x_{3}, x_{4}\right)=\sum_{\sigma \in \mathcal{S}_{3}}(-1)^{\sigma}( & \alpha x_{\sigma(1)} x_{\sigma(2)} x_{\sigma(3)} y+\beta x_{\sigma(1)} x_{\sigma(2)} y x_{\sigma(3)} \\
& \left.+\gamma x_{\sigma(1)} y x_{\sigma(2)} x_{\sigma(3)}+\delta y x_{\sigma(1)} x_{\sigma(2)} x_{\sigma(3)}\right)
\end{aligned}
$$

with $x_{i} \in K_{3}, y \in H_{3}$. Letting $y=e_{11}+e_{22}+e_{33}$, we get $\alpha+\beta+\gamma+\delta=0$, and the substitution $x_{1}=e_{12}-e_{21}, x_{2}=e_{23}-e_{32}, x_{3}=e_{13}-e_{31}, y=e_{11}$ provides the relations $\delta=-\alpha$ and $\gamma=-\beta$ (char $F \neq 2)$. Thus,

$$
f\left(x_{1}, x_{2}, x_{3}, x_{4}\right)=\alpha r\left(x_{1}, x_{2}, x_{3}, x_{4}\right)+\beta\left[r\left(x_{1}, x_{2}, x_{3}, x_{4}\right)-\mathrm{S}_{4}\left(x_{1}, x_{2}, x_{3}, x_{4}\right)\right] .
$$

Now if we let

$$
h\left(x_{1}, x_{2}, x_{3}, x_{4}\right)=\sum_{(123)} g\left(x_{\sigma(1)}, x_{\sigma(2)}, x_{\sigma(3)}, x_{\sigma(4)}\right),
$$

it suffices to notice that $h-r=r-\mathrm{S}_{4}$ to conclude that $f$ is a consequence of $g$ and $r$. We have established

Theorem 3.5. Under the hypotheses of (3.1) on the field F, any polynomial identity of $K_{3}(F, t)$ of minimal degree is a consequence of

$$
\begin{aligned}
& p\left(x_{1}, x_{2}, x_{3}, x_{4}\right)=\sum_{(123)}\left\{x_{1}\left[x_{2}, x_{4}\right] x_{3}\right\}, \\
& q\left(x_{1}, x_{2}, x_{3}, x_{4}\right)=\sum_{(123)}\left\{x_{1}\left[x_{2}, x_{3}\right] x_{4}\right\}+\sum_{(124)}\left\{x_{1}\left[x_{2}, x_{4}\right] x_{3}\right\} \\
& +2\left(\left[x_{1}, x_{3}\right] \mathrm{D}_{x_{2}, x_{4}}+\left[x_{1}, x_{4}\right] \mathrm{D}_{x_{2}, x_{3}}\right. \\
& \left.-\left[x_{2}, x_{3}\right] \mathrm{D}_{x_{1}, x_{4}}-\left[x_{2}, x_{4}\right] \mathrm{D}_{x_{1}, x_{3}}\right), \\
& r\left(x_{1}, x_{2}, x_{3}, x_{4}\right)=\left[\mathrm{S}_{3}\left(x_{1}, x_{2}, x_{3}\right), x_{4}\right] \text {, }
\end{aligned}
$$


and any *-polynomial identity of $\left(M_{3}(F), t\right)$ of minimal degree is a consequence of

$$
\begin{aligned}
& p\left(x_{1}-x_{1}^{*}, x_{2}-x_{2}^{*}, x_{3}-x_{3}^{*}, x_{4}-x_{4}^{*}\right), q\left(x_{1}-x_{1}^{*}, x_{2}-x_{2}^{*}, x_{3}-x_{3}^{*}, x_{4}-x_{4}^{*}\right), \\
& r\left(x_{1}-x_{1}^{*}, x_{2}-x_{2}^{*}, x_{3}-x_{3}^{*}, x_{4} \pm x_{4}^{*}\right), g\left(x_{1}-x_{1}^{*}, x_{2}-x_{2}^{*}, x_{3}-x_{3}^{*}, x_{4}+x_{4}^{*}\right),
\end{aligned}
$$

where $g\left(x_{1}, x_{2}, x_{3}, x_{4}\right)=\sum_{\sigma \in \mathcal{S}_{2}}(-1)^{\sigma}\left(\left\{x_{\sigma(1)} x_{\sigma(2)}\left(x_{3} \circ x_{4}\right)\right\}\right.$.

We move on to $n=4$. The following lemma shows that the only $(4,5, s)$-identities are the identities of $K_{4}$.

Lemma 3.6. Over a field of characteristic not 2, $\left(M_{n}(F), t\right)$ has no $(n, n+1,1)$ identities when $n>3$.

Proof. Write $f\left(x_{1}, \ldots, x_{n+1}\right)=B_{1}+B_{2}+\cdots+B_{n}+B_{n+1}$, where

$$
B_{i}=\sum_{\sigma \in \mathcal{S}_{n}} \alpha_{\sigma}^{i} x_{\sigma(1)} \ldots x_{\sigma(i-1)} x_{n+1} x_{\sigma(i)} \ldots x_{\sigma(n)}, \quad \alpha_{\sigma}^{i} \in F .
$$

First observe that if we can show by way of substitutions that $B_{i}$ is the zero polynomial for $1 \leq i \leq[(n+1) / 2]$ (where $[(n+1) / 2]$ is the largest integer in $(n+1) / 2)$, then taking the transpose of these substitutions yields $B_{n+2-i}$ is identically zero; therefore it suffices to establish that $B_{i} \equiv 0$ for $i=1, \ldots,[(n+1) / 2]$, and for $i=(n+2) / 2$, when $n$ is even.

We first handle the case $n \geq 6$. Fix $i \in\{1,2, \ldots,[(n+1) / 2]\}$. Substituting $\left(e_{12}-\right.$ $\left.e_{21}\right),\left(e_{23}-e_{32}\right), \ldots,\left(e_{i-1 i}-e_{i i-1}\right), e_{i i},\left(e_{i i+1}-e_{i+1 i}\right), \ldots,\left(e_{n-3 n-2}-e_{n-2 n-3}\right)$, for $x_{\sigma(1)}, x_{\sigma(2)}, \ldots, x_{\sigma(i-1)}, x_{n+1}, x_{\sigma(i)}, \ldots, x_{\sigma(n-3)}$, while successively choosing

$$
\begin{aligned}
& \text { 1) }\left(e_{n-2 n-1}-e_{n-1 n-2}\right),\left(e_{n-1 n}-e_{n n-1}\right),\left(e_{n n-1}-e_{n-1 n}\right), \\
& 2) \quad\left(e_{n-2 n-1}-e_{n-1 n-2}\right),\left(e_{n-1 n-2}-e_{n-2 n-1}\right),\left(e_{n-2 n}-e_{n n-2}\right), \\
& 3) \quad\left(e_{n-2 n-1}-e_{n-1 n-2}\right),\left(e_{n-1 n}-e_{n n-1}\right),\left(e_{n n-2}-e_{n-2 n}\right),
\end{aligned}
$$

for $x_{\sigma(n-2)}, x_{\sigma(n-1)}, x_{\sigma(n)}$, yields

$$
\alpha_{\sigma}^{i}=-\alpha_{\sigma \tau_{1}}^{i}=-\alpha_{\sigma \tau_{2}}^{i}=\alpha_{\sigma \tau_{3}}^{i},
$$

where $\tau_{1}=(n-1 n), \tau_{2}=(n-2 n-1)$ and $\tau_{3}=(n-2 n), \sigma \in \mathcal{S}_{n}$. Since $\tau_{1}=\tau_{2} \tau_{3} \tau_{2}$, we have $\alpha_{\sigma}^{i}=0$. If $n$ is odd, we now have $f \equiv 0$; if $n$ is even, we are left with $f=B_{k}, k=(n+2) / 2$. But the substitution $\left(e_{12}-e_{21}\right), \ldots,\left(e_{k-1 k}-e_{k k-1}\right), e_{k k}$, $\left(e_{k k-2}-e_{k-2 k}\right),\left(e_{k-2 k+1}-e_{k+1 k-2}\right),\left(e_{k+1 k+2}-e_{k+2 k+1}\right), \ldots,\left(e_{n-1 n}-e_{n n-1}\right)$, for $x_{\sigma(1)}, \ldots, x_{\sigma(k-1)}, x_{n+1}, x_{\sigma(k)}, x_{\sigma(k+1)}, x_{\sigma(k+2)}, \ldots, x_{\sigma(n)}$ forces $\alpha_{\sigma}^{k}=\alpha_{\sigma \tau}^{k-1}=0$, where $\tau=(k-2 k)$, so again $f \equiv 0$.

The cases $n=4$ or 5 are handled in the same way except for $i=2$ when $n=4$ and $i=3$ when $n=5$. For those, substitutions 2) and 3) should be replaced by

$$
\begin{aligned}
& \left.2^{\prime}\right) \quad\left(e_{12}-e_{21}\right),\left(e_{23}+e_{32}\right),\left(e_{34}-e_{43}\right),\left(e_{43}-e_{34}\right),\left(e_{32}-e_{23}\right), \\
& \left.2^{\prime \prime}\right) \quad\left(e_{12}-e_{21}\right),\left(e_{23}-e_{32}\right),\left(e_{34}+e_{43}\right),\left(e_{45}-e_{54}\right),\left(e_{54}-e_{45}\right),\left(e_{43}-e_{34}\right),
\end{aligned}
$$

which imply

$$
\alpha_{\sigma}^{i}+\alpha_{\sigma \tau_{1}}^{i}-\alpha_{\sigma \tau_{2}}^{n+1}-\alpha_{\sigma \tau_{3}}^{n+1}=0
$$

where $\tau_{1}=(n-2 n-1), \tau_{2}=(n-2 n n-1)$ and $\tau_{3}=(n-2 n)$, and

$$
\begin{aligned}
& \left.3^{\prime}\right) \quad\left(e_{12}-e_{21}\right), e_{22},\left(e_{24}-e_{42}\right),\left(e_{43}-e_{34}\right),\left(e_{32}-e_{23}\right), \\
& \left.3^{\prime \prime}\right) \quad\left(e_{12}-e_{21}\right),\left(e_{23}-e_{32}\right), e_{33},\left(e_{35}-e_{53}\right),\left(e_{54}-e_{45}\right),\left(e_{43}-e_{34}\right),
\end{aligned}
$$


which in turn imply

$$
\alpha_{\sigma}^{i}-\alpha_{\sigma \tau}^{i}+\alpha_{\sigma}^{n+1}-\alpha_{\sigma \tau}^{n+1}=0,
$$

where $\tau=(n-2 n-1)$.

The substitution $\left(e_{12}-e_{21}\right),\left(e_{23}+e_{32}\right),\left(e_{34}-e_{43}\right),\left(e_{43}-e_{34}\right),\left(e_{32}-e_{23}\right)$, for $x_{\sigma(1)}, x_{5}, x_{\sigma(2)}, x_{\sigma(3)}, x_{\sigma(4)}$, implies $\alpha_{\sigma}^{2}+\alpha_{\sigma \tau_{1}}^{2}-\alpha_{\sigma \tau_{2}}^{5}-\alpha_{\sigma \tau_{3}}^{5}=0$, where $\tau_{1}=(23)$, $\tau_{2}=(243)$ and $\tau_{3}=(24)$; thus $\alpha_{\sigma}^{2}=-\alpha_{\sigma \tau_{1}}^{2}$. Finally, the substitution $\left(e_{12}-e_{21}\right)$, $\left(e_{23}-e_{32}\right),\left(e_{34}-e_{43}\right),\left(e_{45}-e_{54}\right),\left(e_{54}-e_{45}\right),\left(e_{43}-e_{34}\right)$ for $x_{\sigma(1)}, x_{\sigma(2)}, x_{6}, x_{\sigma(3)}$, $x_{\sigma(4)}, x_{\sigma(5)}$ yields $\alpha_{\sigma}^{3}+\alpha_{\sigma \tau_{1}}^{3}-\alpha_{\sigma \tau_{2}}^{6}-\alpha_{\sigma \tau_{3}}^{6}=0$, where $\tau_{1}=(34), \tau_{2}=(354)$ and $\tau_{3}=(35) ;$ so $\alpha_{\sigma}^{2}=-\alpha_{\sigma \tau_{1}}^{2}$.

We now show

Theorem 3.7. Under the hypotheses of (3.1) on the field F, any polynomial identity of $K_{4}(F, t)$ of minimal degree is a consequence of

$$
\kappa\left(x_{1}, x_{2}, x_{3}, y\right):=y^{2} \mathrm{~S}_{3}\left(\mathrm{~V}_{x_{1}}, \mathrm{~V}_{x_{2}}, \mathrm{~V}_{x_{3}}\right)-\left(y \mathrm{~S}_{3}\left(\mathrm{~V}_{x_{1}}, \mathrm{~V}_{x_{2}}, \mathrm{~V}_{x_{3}}\right)\right) \circ y
$$

and any *-polynomial identity of $\left(M_{4}(F), t\right)$ of minimal degree is a consequence of $\kappa\left(x_{1}-x_{1}^{*}, x_{2}-x_{2}^{*}, x_{3}-x_{3}^{*}, y-y^{*}\right)$.

Proof. It is obvious that $f(x)=x^{5}$ isn't an identity, and by Kostant-Rowen, $K_{4}$ does not satisfy the standard polynomial $S_{5}$. This takes care of types [5] and $[1,1,1,1,1]$. Consider now an arbitrary polynomial of type $[4,1]$

$$
f(x, y)=\alpha_{1} x^{4} y+\alpha_{2} x^{3} y x+\alpha_{3} x^{2} y x^{2}+\alpha_{4} x y x^{3}+\alpha_{5} y x^{4} .
$$

The coefficients of $e_{13}, e_{31}$ and $e_{12}$ in $f\left(e_{12}-e_{21}, e_{12}-e_{21}+e_{13}-e_{31}+e_{14}-e_{41}\right)$ yield $\alpha_{1}=\alpha_{5}=0$ and $\alpha_{2}+\alpha_{3}+\alpha_{4}=0$; then the coefficients of $e_{12}, e_{14}$ and $e_{21}$ in $f\left(e_{12}-e_{21}+e_{23}-e_{32}+e_{24}-e_{42}, e_{14}-e_{41}\right)$ lead to $\alpha_{2}=\alpha_{3}=\alpha_{4}=0$.

Next, an arbitrary $(4,5,0)$-identity of type [3,2] would have the form

$$
\begin{aligned}
f(x, y)=\alpha_{1} y^{2} x^{3} & +\alpha_{2} y x y x^{2}+\alpha_{3} y x^{2} y x+\alpha_{4} y x^{3} y+\alpha_{5} x y^{2} x^{2} \\
& +\alpha_{6} x y x y x+\alpha_{7} x y x^{2} y+\alpha_{8} x^{2} y^{2} x+\alpha_{9} x^{2} y x y+\alpha_{10} x^{3} y^{2} .
\end{aligned}
$$

We successively compute

$$
\begin{aligned}
& f\left(e_{12}-e_{21}+e_{13}-e_{31}, e_{24}-e_{42}\right) \\
& \quad=\left(\alpha_{5}+2 \alpha_{10}\right) e_{12}+\alpha_{5} e_{13}+\left(-2 \alpha_{1}-\alpha_{8}\right) e_{21}-\alpha_{8} e_{31},
\end{aligned}
$$

which produces $\alpha_{1}=\alpha_{5}=\alpha_{8}=\alpha_{10}=0$; then

$$
\begin{aligned}
& f\left(e_{13}-e_{31}+e_{24}-e_{42}, e_{12}-e_{21}\right) \\
& \quad=\left(\alpha_{1}+\alpha_{3}+\alpha_{8}\right)\left(e_{13}+e_{24}\right)+\left(-\alpha_{5}-\alpha_{7}-\alpha_{10}\right)\left(e_{31}+e_{42}\right)
\end{aligned}
$$

which yields $\alpha_{3}=\alpha_{7}=0$; and finally

$$
\begin{aligned}
f\left(e_{13}-\right. & \left.e_{31}+e_{23}-e_{32}, e_{13}-e_{31}+e_{14}-e_{41}\right) \\
= & \left(4 \alpha_{1}+2 \alpha_{2}+2 \alpha_{3}+2 \alpha_{4}+2 \alpha_{5}+\alpha_{6}+\alpha_{7}+2 \alpha_{8}+\alpha_{9}+2 \alpha_{10}\right) e_{13} \\
& +\left(2 \alpha_{4}+\alpha_{7}+\alpha_{9}+2 \alpha_{10}\right) e_{14} \\
& +\left(2 \alpha_{5}+\alpha_{6}+\alpha_{7}+2 \alpha_{8}+\alpha_{9}+2 \alpha_{10}\right) e_{23}+\left(\alpha_{7}+\alpha_{9}+2 \alpha_{10}\right) e_{24} \\
& -\left(2 \alpha_{1}+\alpha_{2}+\alpha_{3}+2 \alpha_{4}+2 \alpha_{5}+\alpha_{6}+2 \alpha_{7}+2 \alpha_{8}+2 \alpha_{9}+4 \alpha_{10}\right) e_{31} \\
& -\left(2 \alpha_{1}+\alpha_{2}+\alpha_{3}+2 \alpha_{5}+\alpha_{6}+2 \alpha_{8}\right) e_{32}-\left(2 \alpha_{1}+\alpha_{2}+\alpha_{3}+2 \alpha_{4}\right) e_{41} \\
& -\left(2 \alpha_{1}+\alpha_{2}+\alpha_{3}\right) e_{42},
\end{aligned}
$$


in which the coefficients of $e_{42}, e_{24}, e_{23}$ and $e_{41}$ imply that $\alpha_{2}=\alpha_{9}=\alpha_{6}=\alpha_{4}=0$. This shows that $K_{4}$ has no identity of type [3,2].

Next, we treat the case $[3,1,1]$. By $(3.1)$, it suffices to look at polynomials of the form

$$
\begin{aligned}
f\left(x, y_{1}, y_{2}\right) & =\alpha_{1}\left(x^{3} y_{1} y_{2}-x^{3} y_{2} y_{1}\right)+\alpha_{2}\left(x^{2} y_{1} x y_{2}-x^{2} y_{2} x y_{1}\right) \\
& +\alpha_{3}\left(x^{2} y_{1} y_{2} x-x^{2} y_{2} y_{1} x\right)+\alpha_{4}\left(x y_{1} x^{2} y_{2}-x y_{2} x^{2} y_{1}\right) \\
& +\alpha_{5}\left(x y_{1} x y_{2} x-x y_{2} x y_{1} x\right)+\alpha_{6}\left(x y_{1} y_{2} x^{2}-x y_{2} y_{1} x^{2}\right) \\
& +\alpha_{7}\left(y_{1} x^{3} y_{2}-y_{2} x^{3} y_{1}\right)+\alpha_{8}\left(y_{1} x^{2} y_{2} x-y_{2} x^{2} y_{1} x\right) \\
& +\alpha_{9}\left(y_{1} x y_{2} x^{2}-y_{2} x y_{1} x^{2}\right)+\alpha_{10}\left(y_{1} y_{2} x^{3}-y_{2} y_{1} x^{3}\right) .
\end{aligned}
$$

Once again, we use three substitutions:

$$
f\left(e_{12}-e_{21}, e_{23}-e_{32}, e_{34}-e_{43}\right)=-\alpha_{1} e_{14}-\alpha_{10} e_{41},
$$

from which we deduce that $\alpha_{1}=\alpha_{10}=0$;

$$
\begin{aligned}
f\left(e_{12}-\right. & \left.e_{21}+e_{13}-e_{31}, e_{12}-e_{21}, e_{23}-e_{32}\right) \\
& =2\left(\alpha_{1}+\alpha_{3}+\alpha_{6}+\alpha_{10}\right) e_{11}-\left(\alpha_{2}-\alpha_{3}+\alpha_{4}-\alpha_{6}+4 \alpha_{7}+\alpha_{8}+\alpha_{9}\right) e_{22} \\
& +\left(2 \alpha_{1}+\alpha_{2}+\alpha_{3}+\alpha_{4}+\alpha_{6}+2 \alpha_{7}-\alpha_{8}-\alpha_{9}\right) e_{23} \\
& +\left(-\alpha_{2}+\alpha_{3}-\alpha_{4}+\alpha_{6}+2 \alpha_{7}+\alpha_{8}+\alpha_{9}+2 \alpha_{10}\right) e_{32} \\
& +\left(2 \alpha_{1}+\alpha_{2}+\alpha_{3}+\alpha_{4}+\alpha_{6}+\alpha_{8}+\alpha_{9}+2 \alpha_{10}\right) e_{33},
\end{aligned}
$$

which implies that $\alpha_{7}=0, \alpha_{8}=-\alpha_{9}$ and $\alpha_{2}=-\alpha_{4}$; and also

$$
\begin{aligned}
f\left(e_{12}\right. & \left.-e_{21}+e_{34}-e_{43}, e_{23}-e_{32}+e_{24}-e_{42}+e_{34}-e_{43}, e_{13}-e_{31}\right) \\
& =\left(\alpha_{1}+\alpha_{3}+\alpha_{4}+\alpha_{6}+\alpha_{8}+\alpha_{10}\right)\left(e_{11}+e_{22}\right) \\
& -\left(\alpha_{2}-\alpha_{5}+\alpha_{7}+\alpha_{9}\right)\left(e_{12}+e_{21}+e_{34}+e_{43}\right) \\
& -\left(\alpha_{2}+\alpha_{3}+\alpha_{7}+\alpha_{8}+\alpha_{9}+\alpha_{10}\right) e_{13} \\
& -\left(\alpha_{1}+\alpha_{4}+\alpha_{5}+\alpha_{6}\right) e_{24}-\left(\alpha_{1}+\alpha_{2}+\alpha_{4}+\alpha_{6}+\alpha_{7}+\alpha_{9}\right) e_{31} \\
& -2\left(\alpha_{2}+\alpha_{7}+\alpha_{9}\right) e_{33}-\left(\alpha_{3}+\alpha_{5}+\alpha_{8}+\alpha_{10}\right) e_{42}-2 \alpha_{5} e_{44},
\end{aligned}
$$

which implies that $\alpha_{4}=-\alpha_{8}, \alpha_{2}=-\alpha_{9}, \alpha_{5}=0$ and $\alpha_{4}=-\alpha_{6}$. These relations then force $f$ to be a scalar multiple of the polynomial

$$
\begin{aligned}
\tilde{f}(x, y, z)= & x^{2}(y z-z y) x-x(y z-z y) x^{2}-x^{2} y x z+x^{2} z x y+x y x^{2} z \\
& -x z x^{2} y-y x^{2} z x+z x^{2} y x+y x z x^{2}-z x y x^{2} \\
= & (\{x y z\}-\{x z y\}) U_{x}+z U_{x} U_{x, y}-\left\{y x z U_{x}\right\}-y U_{x} U_{x, z} \\
& +\left\{z x y U_{x}\right\} .
\end{aligned}
$$

Now it suffices to notice from $(2.23)$ that $\tilde{f}(x, y, z)=\kappa(z, y, x, x)$, that is, $f$ is a consequence of our polynomial $\kappa$.

We continue with an arbitrary polynomial of type $[2,2,1]$; using $(3.1)$, it has the form

$$
\begin{aligned}
& f(x, y, z)=\alpha_{1}\left(x^{2} y^{2} z+y^{2} x^{2} z\right)+\alpha_{2}\left(x^{2} y z y+y^{2} x z x\right)+\alpha_{3}\left(x^{2} z y^{2}+y^{2} z x^{2}\right) \\
& \quad+\alpha_{4}\left(x z x y^{2}+y z y x^{2}\right)+\alpha_{5}\left(z x^{2} y^{2}+z y^{2} x^{2}\right)+\alpha_{6}(x y x y z+y x y x z) \\
& \quad+\alpha_{7}(x y x z y+y x y z x)+\alpha_{8}(x y z x y+y x z y x)+\alpha_{9}(x z y x y+y z x y x) \\
& \quad+\alpha_{10}(z x y x y+z y x y x) .
\end{aligned}
$$


We first compute the substitution

$$
\begin{aligned}
& f\left(e_{12}-e_{21}, e_{13}-e_{31}, e_{12}-e_{21}+e_{13}-e_{31}+e_{14}-e_{41}\right)=2 \alpha_{1} e_{14}-2 \alpha_{5} e_{41} \\
& +\left(2 \alpha_{1}+\alpha_{2}+\alpha_{3}\right) e_{12}-\left(\alpha_{3}+\alpha_{4}+2 \alpha_{5}\right) e_{21}+\ldots,
\end{aligned}
$$

from which we get $\alpha_{1}=\alpha_{5}=0$ and $\alpha_{2}=\alpha_{4}=-\alpha_{3}$. Then the substitution

$$
\begin{aligned}
& f\left(e_{12}-e_{21}+e_{13}-e_{31}+e_{14}-e_{41}, e_{12}-e_{21}+e_{34}-e_{43}, e_{23}-e_{32}\right) \\
& \quad=-\left(\alpha_{4}+\alpha_{6}+\alpha_{8}+\alpha_{9}\right) e_{12}-\left(\alpha_{4}+\alpha_{9}\right) e_{13}-\left(3 \alpha_{2}+\alpha_{4}+\alpha_{7}-\alpha_{9}\right) e_{14} \\
& \quad+\left(\alpha_{2}+\alpha_{7}+\alpha_{8}+\alpha_{10}\right) e_{21}-\left(2 \alpha_{1}-2 \alpha_{5}+\alpha_{6}-\alpha_{10}\right) e_{22} \\
& \quad+\left(2 \alpha_{1}+2 \alpha_{3}+2 \alpha_{5}+2 \alpha_{6}-\alpha_{8}\right) e_{23}+\left(\alpha_{3}+2 \alpha_{5}-\alpha_{8}\right) e_{24} \\
& \quad+\left(\alpha_{2}+\alpha_{7}\right) e_{31}-\left(2 \alpha_{1}+2 \alpha_{3}+2 \alpha_{5}-\alpha_{8}+2 \alpha_{10}\right) e_{32} \\
& \quad+\left(2 \alpha_{1}-2 \alpha_{5}+\alpha_{6}-\alpha_{10}\right) e_{33}-\left(\alpha_{3}+2 \alpha_{5}+\alpha_{10}\right) e_{34} \\
& \quad+\left(\alpha_{2}+3 \alpha_{4}-\alpha_{7}+\alpha_{9}\right) e_{41}-\left(2 \alpha_{1}+\alpha_{3}-\alpha_{8}\right) e_{42} \\
& \quad+\left(2 \alpha_{1}+\alpha_{3}+\alpha_{6}\right) e_{43}
\end{aligned}
$$

implies that all remaining $\alpha_{i}$ 's must vanish. Therefore, there are no identities of type $[2,2,1]$.

The only remaining case is type $[2,1,1,1]$. A typical polynomial looks like

$$
\begin{aligned}
f\left(x_{1}, x_{2}, x_{3}, y\right)= & \sum_{\sigma \in \mathcal{S}_{3}}\left(\alpha_{1} x_{\sigma(1)} x_{\sigma(2)} x_{\sigma(3)} y^{2}+\alpha_{2} x_{\sigma(1)} x_{\sigma(2)} y x_{\sigma(3)} y\right. \\
& +\alpha_{3} x_{\sigma(1)} y x_{\sigma(2)} x_{\sigma(3)} y+\alpha_{4} y x_{\sigma(1)} x_{\sigma(2)} x_{\sigma(3)} y+\alpha_{5} x_{\sigma(1)} x_{\sigma(2)} y^{2} x_{\sigma(3)} \\
& +\alpha_{6} x_{\sigma(1)} y x_{\sigma(2)} y x_{\sigma(3)}+\alpha_{7} y x_{\sigma(1)} x_{\sigma(2)} y x_{\sigma(3)}+\alpha_{8} x_{\sigma(1)} y^{2} x_{\sigma(2)} x_{\sigma(3)} \\
& \left.+\alpha_{9} y x_{\sigma(1)} y x_{\sigma(2)} x_{\sigma(3)}+\alpha_{10} y^{2} x_{\sigma(1)} x_{\sigma(2)} x_{\sigma(3)}\right) .
\end{aligned}
$$

From the substitution

$$
\begin{aligned}
f\left(e_{12}\right. & \left.-e_{21}+e_{13}-e_{31}, e_{23}-e_{32}, e_{34}-e_{43}, e_{12}-e_{21}+e_{23}-e_{32}+e_{34}-e_{43}\right) \\
= & \left(\alpha_{2}+\alpha_{3}+\alpha_{7}+\alpha_{9}\right) e_{11}-\left(\alpha_{5}+\alpha_{6}-\alpha_{9}-\alpha_{10}\right)\left(e_{12}+e_{43}\right) \\
& -\left(\alpha_{1}+\alpha_{2}+2 \alpha_{5}+\alpha_{6}+2 \alpha_{8}+\alpha_{9}+\alpha_{10}\right)\left(e_{14}+e_{41}\right) \\
& +\left(\alpha_{1}+\alpha_{2}-\alpha_{6}-\alpha_{8}\right)\left(e_{21}+e_{34}\right)+\left(\alpha_{2}+\alpha_{3}+\alpha_{7}+\alpha_{9}\right) e_{22} \\
& +\left(\alpha_{3}+\alpha_{4}+\alpha_{6}+\alpha_{7}\right)\left(e_{23}+e_{32}\right)-\left(\alpha_{2}-\alpha_{3}+\alpha_{5}-\alpha_{8}\right) e_{24} \\
& +\left(2 \alpha_{3}+4 \alpha_{6}+2 \alpha_{7}\right) e_{33}+\left(\alpha_{5}+\alpha_{7}-\alpha_{8}-\alpha_{9}\right) e_{42}-2\left(\alpha_{2}+\alpha_{9}\right) e_{44}
\end{aligned}
$$

we deduce the relations $\alpha_{1}=-\alpha_{10}, \alpha_{2}=-\alpha_{9}, \alpha_{3}=-\alpha_{7}, \alpha_{4}=-\alpha_{6}, \alpha_{5}=-\alpha_{8}$, $\alpha_{6}=0, \alpha_{1}+\alpha_{2}-\alpha_{8}=0$ and $\alpha_{1}+\alpha_{3}+\alpha_{8}=0$. The dimension of the nullspace is 2 , and we let $\alpha_{1}$ and $\alpha_{8}$ play the role of free variables.

We first set $\alpha_{1}=\alpha_{8}=1$ : then $\alpha_{2}=\alpha_{4}=\alpha_{6}=\alpha_{9}=0, \alpha_{3}=-2, \alpha_{5}=-1$, $\alpha_{7}=2, \alpha_{10}=-1$, and we get

$$
\begin{aligned}
f_{1}\left(x_{1}, x_{2}, x_{3}, y\right)= & \sum_{\sigma \in \mathcal{S}_{3}}(-1)^{\sigma}\left(x_{\sigma(1)} x_{\sigma(2)} x_{\sigma(3)} y^{2}-2\left(x_{\sigma(1)} y x_{\sigma(2)} x_{\sigma(3)} y\right.\right. \\
& \left.-y x_{\sigma(1)} x_{\sigma(2)} y x_{\sigma(3)}\right)-x_{\sigma(1)} x_{\sigma(2)} y^{2} x_{\sigma(3)} \\
& \left.+x_{\sigma(1)} y^{2} x_{\sigma(2)} x_{\sigma(3)}-y^{2} x_{\sigma(1)} x_{\sigma(2)} x_{\sigma(3)}\right)
\end{aligned}
$$

but it is easily seen that $f_{1}\left(x_{1}, x_{2}, y, y\right)=\kappa\left(x_{1}, x_{2}, y, y\right)-2 q\left(y, x_{1}, x_{2}\right)$. 
Next we let $\alpha_{1}=0, \alpha_{8}=1$; then $\alpha_{4}=\alpha_{6}=\alpha_{10}=0$ while $\alpha_{3}=\alpha_{5}=\alpha_{9}=-1$ and $\alpha_{2}=\alpha_{7}=1$, and we get

$$
\begin{aligned}
f_{2}\left(x_{1}, x_{2}, x_{3}, y\right)= & \sum_{\sigma \in \mathcal{S}_{3}}(-1)^{\sigma}\left(x_{\sigma(1)} x_{\sigma(2)} y x_{\sigma(3)} y\right. \\
& -x_{\sigma(1)} x_{\sigma(2)} y^{2} x_{\sigma(3)}-x_{\sigma(1)} y x_{\sigma(2)} x_{\sigma(3)} y+x_{\sigma(1)} y^{2} x_{\sigma(2)} x_{\sigma(3)} \\
& \left.+y x_{\sigma(1)} x_{\sigma(2)} y x_{\sigma(3)}-y x_{\sigma(1)} y x_{\sigma(2)} x_{\sigma(3)}\right)
\end{aligned}
$$

and, from $(2.23)$,

$$
f_{2}\left(x_{1}, x_{2}, x_{3}, y\right)=\kappa\left(x_{1}, x_{2}, x_{3}, y\right) .
$$

Thus, any $[2,1,1,1]$-identity, and in fact any identity of $K_{4}(F)$, is a consequence of our polynomial $\kappa$.

In a future paper, we will present the minimal degree $*$-identities of $\left(M_{n}(F), s\right)$, $n<5$, for $s$ the symplectic involution. For the transpose involution, the minimal identities involve mostly skew-symmetric variables; naturally, when $*=s$, it appears that most variables will be symmetric. But regardless of the involution, we do not expect the surprising richness of $*$-identities that occurred for low $n$ 's to carry over to the general case $n \geq 5$.

\section{REFERENCES}

1. Amitsur, A. S. and Levitzki, J., Minimal identities for algebras, Proc. Amer. Math. Soc. 1 (1950), 449-463. MR 12:155d

2. Amitsur, A. S., Identities in rings with involution, Israel J. Math. 7 (1969), 63-68. MR 39: 4216

3. Drensky, Vesselin, and Giambruno, Antonio, Cocharacters, codimensions and Hilbert series of the polynomial identities for $2 \times 2$ matrices with involution, Canad. J. Math. 46 (1994), 718-733. MR 95d:16028

4. Giambruno, Antonio, On *-polynomial identities for $n \times n$ matrices, J. Algebra 133 (1990) 433-438. MR 91g:16015

5. Kostant, B., A theorem of Frobenius, a theorem of Amitsur-Levitzki, and cohomology theory, Indiana J. Math. 7 (1958), 237-264. MR 19:1153e

6. Kostant, B., A Lie algebra generalization of the Amitsur-Levitzki theorem, Adv. in Math. 40 (1980), 155-175. MR 82h:17008

7. Levchenko, Diana V., Finite basis property of identities with involution of a second-order matrix algebra (Russian), Serdica 8 (1982), 42-56. MR 84e:16008

8. Levchenko, Diana V., Identity with involution of a second-order matrix algebra (Russian), C.R. Acad. Bulgare Sci. 33 (1980), 1043-1045. MR 82h:16011

9. Ma, W., A decomposition of elements of the free algebra, Proc. Amer. Math. Soc. 118 (1993), 37-45. MR 93g: 16040

10. Ma, W., Racine, M. L., Minimal identities of symmetric matrices, Trans. Amer. Math. Soc. 320 (1990), 171-192. MR 91k:16018

11. Neher, E., Jordan Triple Systems by the Grid Approach, Lecture Notes in Mathematics 1280, Springer, New York, 193 pp. MR 89b:17024

12. Racine, M. L., Central polynomials for Jordan algebras I, J. Algebra 41 (1976) 224-237. MR 54:5303

13. Racine, M. L., Minimal identities for Jordan algebras of degree 2, Comm. Alg. 5 (1985), 2493-2506. MR 87e:17026

14. Razmyslov, Y. P., Finite basing of the identities of a matrix algebra of second order over a field of characteristic 0, Algebra and Logic 12 (1973), 47-63. MR 49:5103

15. Rowen, L. H., Standard polynomials in matrix algebras, Trans. Amer. Math. Soc. 190 (1974), 253-284. MR 50:2208 
16. Rowen, L. H., Polynomial Identities in Ring Theory, Academic Press, New York, 1980, 365 pp. MR 82a: 16021

17. Rowen, L. H., A simple proof of Kostant's theorem and an analogue for the symplectic involution, Contemp. Math., vol. 13, Amer. Math. Soc. (1982), 207-215. MR 84d:16024

Department of Mathematics \& Computer Science, Denison University, Granville, Оніо 43023

E-mail address: damour@cc.denison.edu

Department of Mathematics, University of Ottawa, Ottawa, Ontario, K1N 6N5, CANADA

E-mail address: me@mathstat.uottawa.ca 